\title{
Translocation of enterohemorrhagic Escherichia coli effector Tir to the plasma membrane via host Golgi apparatus
}

\author{
CHAN MAO ${ }^{1,2}$, JIANG GU $^{3}$, HAI-GUANG WANG ${ }^{1}$, YAO FANG $^{1}$, PING YANG $^{4}$, \\ BIN TANG ${ }^{1}$, NA LI $^{1}$, TING-TING WANG ${ }^{3}$, QUAN-MING ZOU ${ }^{3}$ and QIAN LI ${ }^{1}$ \\ ${ }^{1}$ Department of Clinical Microbiology and Immunology, Southwest Hospital and College of \\ Medical Laboratory Science; ${ }^{2}$ Department of Pharmacy, Southwest Hospital; ${ }^{3}$ National Engineering Research Center \\ for Immunobiological Products, Department of Microbiology and Biochemical Pharmacy, College of \\ Pharmacy, Third Military Medical University, Chongqing 400038; ${ }^{4}$ Department of Core Facilities of \\ Imaging Facility, Medical College of Zhejiang University, Hangzhou, Zhejiang 310031, P.R. China
}

Received June 17, 2016; Accepted May 31, 2017

DOI: $10.3892 / \mathrm{mmr} .2017 .6763$

\begin{abstract}
The translocated intimin receptor (Tir) is a canonical type III secretion system effector, secreted by the enterohemorrhagic Escherichia coli (E. coli). This receptor alters the regular cellular processing of host cells, to promote intracellular bacterial replication and evasion of the host immune system. Tir is translocated and integrated into the host cell plasma membrane, a process required for its pathogenic activity in these cells, however, the underlying mechanisms of how this occurs remain to be elucidated. The present study used immunofluorescence and immunoelectron microscopy to demonstrate that the Tir of enterohemorrhagic E. coli was localized to the plasma membrane and colocalized with the $58 \mathrm{~K}$ Golgi protein of the host cells. Treatment with brefeldin A destroyed the Golgi structure, inhibited the formation of actin pedestal and blocked the localization of Tir on the host cell plasma membrane. The results of the present study suggested that Tir is translocated to the host plasma membrane in a Golgi-dependent manner. It may mimic the activities of eukaryotic secretory proteins in order to make use of the Golgi apparatus for transportation and integration into the plasma membrane. These findings reveal a novel trafficking pathway
\end{abstract}

Correspondence to: Dr Qian Li, Department of Clinical Microbiology and Immunology, Southwest Hospital and College of Medical Laboratory Science, Third Military Medical University, 30 Gaotanyan Street, Chongqing 400038, P.R. China

E-mail: liqianjane@163.com

Abbreviations: EHEC, Enterohemorrhagic Escherichia coli; EPEC, Enteropathogenic Escherichia coli; Tir, translocated intimin receptor; Golgi, Golgi apparatus; PM, plasma membrane; MOI, multiplicity of infection; A/E lesions, attaching and effacing lesions; BFA, brefeldin A; T3SS, type III secretion system

Key words: actin pedestal, bacterial secretory effectors, enterohemorrhagic Escherichia coli, Golgi apparatus, translocated intimin receptor for the translocation of bacterial secretory effectors to their specific subcellular compartments.

\section{Introduction}

Bacterial effectors are proteins secreted by pathogenic bacteria into their host cells, using a type III, IV, or VI secretion system (T3/4/6SS) (1). These effectors alter the cellular processes to promote intracellular bacterial replication, intercellular spread and evasion of the host immune system (2). To attain full catalytic activity, effectors must be localized in their specific subcellular compartments, such as the nucleus, mitochondria, endoplasmic reticulum (ER), or plasma membrane (PM) (3). However, the mechanisms of translocation of effectors to their target locations are still not well understood.

Bacterial pathogens including enterohemorrhagic Escherichia coli (EHEC) and enteropathogenic Escherichia coli (EPEC) carry a type III secretion system (T3SS) and a number of associated effectors, which contribute to bacterial virulence (4). The attaching and effacing lesions (A/E lesions), which are characterized by accumulation of polymerized actin beneath sites of bacterial attachment, result from the interaction between T3SS effectors and the host proteins (4). Actin pedestals are formed when EHEC or EPEC inject the translocated intimin receptor (Tir) into the host cell PM, where it serves as a bacterial surface receptor for intimin (5). In EHEC, the intimin-Tir interaction is promoted by the Tir cytoskeleton coupled protein $(6,7)$, which recruits the insulin receptor tyrosine kinase substrate p53 (IRSp53), and the actin nucleation-promoting factor Wiskott-Aldrich syndrome protein (N-WASP) (8). However, in EPEC, Tir is phosphorylated, and activates N-WASP with the help of the adapter protein Nck (9). These two pathways generate similar pathological A/E lesions $(10,11)$.

Tir, an essential effector in A/E lesions signaling transduction and a type III transmembrane protein with an approximate molecular weight of $58 \mathrm{kDa}$ (12), is injected into host cells and integrated into the PM. However, the detailed mechanism of Tir transportation and integration into the PM remains unknown. In this study, we investigated the possible pathway(s) 
of Tir transportation. We found that the EHEC Tir colocalizes with the 58K Golgi protein of the host cells. Damage to Golgi structure by brefeldin A (BFA) blocks the localization of Tir on host plasma membrane. Our results suggest that Tir is translocated to plasma membrane through the Golgi apparatus.

\section{Materials and methods}

Cell lines, bacterial strains, and antibodies. HeLa cells were purchased from the American Type Culture Collection (ATCC, CCL-2) and were grown in DMEM (Hyclone, Logan, UT, USA) supplemented with $10 \%$ fetal bovine serum (FBS) (Hyclone) and antibiotics at $37^{\circ} \mathrm{C}$ in a $5 \% \mathrm{CO}_{2}$ atmosphere. The EHEC O157:H7 Sakai strain was obtained from the ATCC (BAA-460). The monoclonal antibody against EspA of EHEC (1H10) was used as a marker for EHEC staining and was produced as described by Yu et al (13). The recombinant carboxyl terminus of the Tir protein, containing residues of Ala405-Ala523 (reTirC $405-523$ ), was produced for the preparation of anti-Tir antibodies. In brief, the gene fragment encoding reTirC ${ }_{405-523}$ was amplified from whole genome of EHEC Sakai (ATCC BAA-460) using primer pairs $\mathrm{P}_{\text {tirC-F }}$ and $\mathrm{P}_{\text {tir } C-R}$ (Table I). The amplified gene fragment was cloned into pET22b vector at NdeI and XhoI restriction sites. The pET22b-reTirC $\mathrm{C}_{405-523}$ was transformed into E. coli BL21 and the recombinant protein was induced by adding of $1 \mathrm{mM}$ of isopropyl $\beta$-D-1-thiogalactopyranoside (IPTG). Cells were then harvested and homogenized in PBS ( $\mathrm{pH}$ 7.2). The soluble fraction was collected and went through a $\mathrm{Ni}^{2+}$-NTA resin (Qiagen) and the His-tagged reTirC $_{405-523}$ was eluted with buffer A containing $250 \mathrm{mM}$ imidazole. Elution from $\mathrm{Ni}^{2+}$-NTA resin was then bound to Resource Q column (GE Healthcare, Piscataway, NJ, USA) and eluted with a gradient concentration buffer (containing PBS and $1 \mathrm{M} \mathrm{NaCl}, \mathrm{pH}$ 7.2). Finally purified reTirC ${ }_{405-523}$ was concentrated and verified by $\mathrm{N}$-terminal sequencing and SDS-PAGE.

For the production of anti-Tir antibodies, purified reTirC $\mathrm{C}_{405-523}$ was formulated with complete or incomplete Freund's Adjuvant as needed (F5881 and F5506; Sigma-Aldrich, St. Louis, MO, USA) and used to immunize rabbits. Then, serum from immunized rabbits was collected and anti-reTirC ${ }_{405-523}$ IgG was purified by affinity chromatography using a protein $\mathrm{G}$ column. The purified rabbit anti-Tir polyclonal antibody was further analyzed by SDS-PAGE, western blotting and immunofluorescence assay.

Construction of $\triangle$ tir EHEC O157 mutants and its complemented mutant. Strain $\Delta$ tir EHEC O157:H7 (tir knockout) was constructed using the $\lambda$ Red recombinase method (14). Primer pairs $\mathrm{P}_{t i r} 1$ and $\mathrm{P}_{t i r} 2$ (Table I) were designed to amplify the chloramphenicol resistance gene from plasmid pKD3 (kindly provided by George F. Gao, Institute of Microbiology, Chinese Academy of Sciences). The purified PCR product was electroporated into $E$. coli $\mathrm{O} 157: \mathrm{H} 7$ carrying the $\lambda$ Red recombinase expression plasmid pKD46 (kindly provided by George F. Gao, Institute of Microbiology, Chinese Academy of Sciences). Primers located inside the chloramphenicol resistance cassette were used to verify deletion of tir by PCR $\left(\mathrm{P}_{t i r} 3\right.$ and $\mathrm{P}_{t i r}$ 4) (Table I). EHEC O157:H7 clones were grown on LB medium containing $25 \mu \mathrm{g} / \mathrm{ml}$ of chloramphenicol to select for chloramphenicol resistance. The pKD46 plasmid was inactivated by growing bacteria at $42^{\circ} \mathrm{C}$.

To construct complementary strains, the gene encoding Tir plus its promoter was amplified using primer pairs $\mathrm{P}_{\text {tir-F }}$ and $\mathrm{P}_{\text {tir-R }}$ (Table I). The gene encoding Tir-HA was amplified by $\mathrm{P}_{\text {tir-F }}$ and $\mathrm{P}_{\text {tirHA-R }}$. They were cloned into pBluescript II SK (-) vector (Agilent Technologies, Inc., CA, USA), and transformed into $\Delta$ tir EHEC O157:H7. The expression of Tir was detected by western blotting using purified rabbit anti-Tir polyclonal antibodies as primary antibody.

Infection of HeLa cells and BFA treatment. HeLa cells were infected with EHEC strains as described by Wang et al (15). with minor modifications. Briefly, HeLa cell monolayers were grown on 20x20 mm coverslips in a 6-well tissue culture plate. Monolayers were co-incubated with wild-type or $\Delta$ tir EHEC O157:H7 Sakai (multiplicity of infection, 100:1) for $5 \mathrm{~h}$ at $37^{\circ} \mathrm{C}$ and $5 \% \mathrm{CO}_{2}$ in the infection medium (2\% FBS DMEM). Additionally, monolayers were pretreated with $2.5 \mu \mathrm{g} / \mathrm{ml}$ of BFA (Beyotime Biotech, Jiangsu, China) for $30 \mathrm{~min}$, prior to EHEC infection (16). At $3 \mathrm{~h}$ post-infection, cells were washed gently twice with phosphate-buffered saline (PBS), followed by addition of an equal volume of fresh infection medium with similar BFA concentration.

Immunofluorescence microscopy. HeLa cells were fixed in $2.5 \%$ paraformaldehyde (Boster, Wuhan, China) and then permeabilized with $0.1 \%$ Triton X-100 (Sigma-Aldrich) with gentle shaking. Permeabilized cells were incubated with rabbit polyclonal anti-Tir-antibody diluted 1:600 in $0.1 \%$ bovine serum albumin (BSA) (Boster Inc., Wuhan, China) in PBS for $1 \mathrm{~h}$, and treated with FITC-labeled goat anti-rabbit secondary antibody (1:500) (Beyotime Biotech) for $1 \mathrm{~h}$. HA-tagged Tir constructs were visualized by staining with a mouse anti-HA antibody (Sigma-Aldrich) followed by Alexa Fluor 633-conjugated goat anti-mouse secondary antibody (Invitrogen Life Technologies, Carlsbad, CA, USA). To visualize Golgi, cells were incubated with mouse anti-58K Golgi protein monoclonal antibody (Abcam, Cambridge, MA, USA) at a dilution of 1:500. Cells were then washed and incubated with Alexa Fluor 633-conjugated goat anti-mouse secondary antibody diluted 1:500 for $1 \mathrm{~h}$.

Polymerized actin was visualized by incubation of cells with FITC-labeled phalloidin (Sigma) diluted 1:200 in PBS for $40 \mathrm{~min}$. The monoclonal antibody $1 \mathrm{H} 10$ was used as a marker for EHEC staining. Cells were stained with $1 \mathrm{H} 10$ diluted at 1:500 for $1 \mathrm{~h}$ followed by incubation with Alexa Fluor 633-conjugated goat anti-mouse antibody as the secondary antibody. All steps were performed at room temperature. Cells were then examined using a fluorescence microscope (Zeiss Axioimager, Oberkochen, Germany). Images were analyzed using LAS AF Lite Leica confocal imaging software (Leica, Heidelberg, Germany).

To quantify the colocalization of Tir and 58K Golgi protein, the colocalization index was calculated by Pearson's correlation with the colocalization finder plugin of ImageJ (US National Institutes of Health, Bethesda, MD, USA). The quantification of actin polymerizations was performed as described previously (9). The number of actin pedestals and attached 
bacterial foci were counted manually in at least 40 fields and used to calculate the percentage of pedestal formation.

Immunoelectron microscopy. The infected HeLa cells were prepared as described above. Subsequently, cells were fixed with $2 \%$ paraformaldehyde and $0.2 \%$ glutaraldehyde in PBS ( $\mathrm{pH}$ 7.4) for $24 \mathrm{~h}$ at $4^{\circ} \mathrm{C}$. Cells were then washed twice with PBS and dehydrated with serial diluted ethanol (30, 50, 70, 90 and $100 \%$ ethanol, $15 \mathrm{~min}$ each). The specimens were embedded in LR-White acrylic resin (SPI Supplies, West Chester, PA, USA). Polymerization of the LR-White was performed at $-20^{\circ} \mathrm{C}$ for $72 \mathrm{~h}$, and at room temperature for $48 \mathrm{~h}$, under ultraviolet light. Primary antibody against Tir was used at a dilution of 1:500. Gold-labeled secondary antibody with a gold particle size of $10 \mathrm{~nm}$ (Sigma) was used at a dilution of 1:30. Sections were observed using a TECNAL-10 transmission electron microscope (Philips Medical Systems B.V., Eindhoven, The Netherlands).

Cellular fractionation and western blotting. Fractionation of HeLa cells was accomplished according to the procedure described previously (17). Briefly, after 5 h of infection, HeLa cells were washed with ice-cold PBS and resuspended in a homogenization buffer ( $3 \mathrm{mM}$ imidazole, $250 \mathrm{mM}$ sucrose, $0.5 \mathrm{mM}$ EDTA, pH 7.4) supplemented with protease inhibitor cocktail (Roche Diagnostics GmbH, Mannheim, Germany). Cells were mechanically disrupted using a grinder. The homogenate was centrifuged at $3,000 \times \mathrm{g}$ for $15 \mathrm{~min}$ at $4^{\circ} \mathrm{C}$ to pellet adherent bacteria, the host nuclei and cytoskeleton. The supernatant was then subjected to high-speed ultracentrifugation $(41,000 \mathrm{x} \mathrm{g})$ for $20 \mathrm{~min}$ at $4^{\circ} \mathrm{C}$ in a $\mathrm{JA}-21$ rotor in a Avanti ${ }^{\circledR} \mathrm{J}-\mathrm{E}$ centrifuge (Beckman Coulter, Inc., Brea, CA, USA) to separate host cell membranes (pellet) from cytoplasm (supernatant). The membrane pellet for alkaline phosphatase treatment was solubilized in $50 \mathrm{mM}$ Tris (pH 8.0)-1\% Triton X-100 without phosphatase inhibitors and incubated with $2 \mathrm{U}$ of alkaline phosphatase (New England Biolabs) for $1 \mathrm{~h}$ at $37^{\circ} \mathrm{C}$ (12). The samples were resuspended in Laemmli sample buffer and boiled for $5 \mathrm{~min}$.

Equal volumes of all fractions were separated using SDS-PAGE, transferred onto PVDF membranes, and probed with anti-Tir, anti-HA (Sigma), anti-Calnexin (Beyotime Biotech) (marker for membrane fraction) and anti-Tubulin (Beyotime Biotech) (marker for cytosol fraction), followed by treatment with the appropriate horseradish peroxidase-conjugated secondary antibodies (Jackson Immunoresearch Laboratories, West Grove, PA, USA). The protein of interest was visualized using Supersignal ${ }^{\circledR}$ West Dura Duration substrate reagent (Thermo Fisher Scientific, Inc., Waltham, MA, USA).

Statistical analysis. The colocalization index and the percentage of pedestal formation were analyzed using ANOVA method from three independent experiments. $\mathrm{P}<0.05$ was considered to indicate a statistically significant difference.

\section{Results}

Tir localizes to host Golgi apparatus. Tir plays an important role in the transportation and modification of proteins in eukaryotic cells (18). In order to observe the subcellular localization of Tir, we generated the rabbit anti-reTirC $\mathrm{C}_{405-523}$ antibody, against residues Ala405-Ala523. The purified rabbit anti-reTirC $\mathrm{C}_{405-523}$ antibody was analyzed by SDS-PAGE (Fig. 1A and B). To confirm the specific binding of anti-reTirC ${ }_{405-523}$ antibody to Tir, we performed the western blotting and immunofluorescence assay, and the effective immune responses were observed (Fig. 1C and D). To better understand the function of the EHEC Tir, we first examined the localization of Tir in the host cells using immunofluorescence microscopy. As shown in Fig. 2A, when HeLa cells were infected with EHEC, Tir was clearly localized inside the host cells surrounding the nuclei. Co-staining with the anti-58K Golgi protein indicated that the two proteins were co-localized extensively.

In addition, we constructed the $\triangle$ tir EHEC deletion and its complemented mutants using the $\lambda$ Red recombinase method (14), and the deletion was confirmed by western blotting (Fig. 1E and F). No apparent Tir staining was observed in HeLa cells that were not infected or infected with EHEC $\Delta t i r$ (Fig. 2A). When EHEC $\Delta$ tir was transformed with the Tir-containing plasmid pBluscript-tir, Tir was detected in HeLa cells similar to the indigenous Tir. The HA-tagged Tir was colocalized with the 58K Golgi protein (Fig. 2B). To further confirm the colocalization of Tir and 58K Golgi protein, the colocalization index was calculated using the Pearson's correlation coefficient. The colocalization index of EHEC-infected cells was significantly higher than that of EHEC $\Delta$ tir infected or uninfected cells $(\mathrm{P}<0.05$, Fig. $2 \mathrm{C})$. The colocalization index of EHEC $\Delta$ tir (+pBluscript-tir) was similar to that of the wild type EHEC (P>0.05; Fig. 2C). However, no colocalization was observed with antibody against Calreticulin (Abcam), a marker for endoplasmic reticulum (data not shown). These results suggest that Tir accumulates in the Golgi complex after the EHEC infection.

The subcellular location of Tir was further analyzed by immunoelectron microscopy. Consistent with immunofluorescence microscopy, our result showed that gold particles were localized on the cell membrane of EHEC-infected HeLa cells, especially at the site of bacterial invasion (Fig. 2D, left). Gold particles were also observed on Golgi structure (Fig. 2D, right). No gold particle was detected in uninfected cells, or cells infected with EHEC $\Delta$ tir (data not shown). Taken together, these results indicate that the EHEC Tir was translocated to the plasma membrane and Golgi body of the host cells.

BFA blocks the formation of actin pedestals. During infection, EHEC injects effector proteins, including Tir, into the host cell to activate the actin cytoskeleton and promote formation of actin pedestals. To further confirm the critical role of Golgi-localized Tir in infection, the formation of actin pedestals was analyzed using BFA, a fungal metabolite that disrupts Golgi structure (16). As shown in Fig. 3A, actin pedestals were clearly observed when host cells were infected with EHEC (upper panel). Treatment with $2.5 \mu \mathrm{g} / \mathrm{ml}$ of BFA, however, revealed few actin pedestals (Fig. 3A, middle panel). BFA did not affect the actin polymerization per se. The large clusters of polymerized actin were counted as pedestals and considered as background (Fig. 3A, lower panel and B). The number of actin pedestals in the absence or presence of BFA 
Table I. Primers used in this study.

\begin{tabular}{lll}
\hline Primers & \multicolumn{1}{c}{ Sequence } & \multicolumn{1}{c}{ Notes } \\
\hline PtirC-F & 5'-CATATGCGTACGGTAGAGAATAAGCCTG-3' & Amplication of tirC405-523 \\
PtirC-R & 5'-CTCGAGCGCCAGACGCGCATAAGTGCTTTG-3' & Amplication of tirC405-523 \\
Ptir1 & 5'-CAATGTGAATAATTCAATTCCTCCTGCACCTC & Knockout of tir \\
& CATTACCTTCACAAACCGTGTGTAGGCTGGAG & \\
Ptir2 & CTGC-3' & Knockout of tir \\
& 5'-CCCGTTAATCCTCCCATGTCATGGCGTAATCCACCA & \\
Ptir3 & CTTAGCGCCAGACGCATATGAATATCCTCCTT-3' & Detection of tir \\
Ptir-F & 5'-ATGCCTATTGGTAATCTTGGTCATA-3' & Detection of tir \\
Ptir-R & 5'-TTAGACGAAACGATGGGATCCCGGCGCT-3' & Amplication of tir and its promoter \\
PtirHA-R & 5'-CGGGGTACCCCGCACGTGGTGTTTACTTTTA-3' & Amplication of tir and its promoter \\
& 5'-CCCAAGCTTGGGTTAAGCGTAGTCTGGGA & Amplication of tir and HA tag \\
& CGTCGTATGGGTAGACGAAACGATGGG-3' & (underlined sequence encodes HA tag)
\end{tabular}
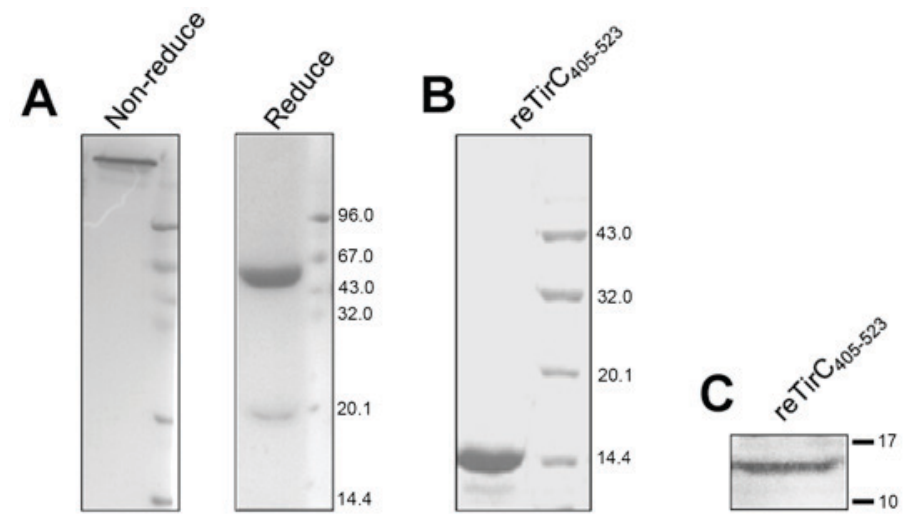

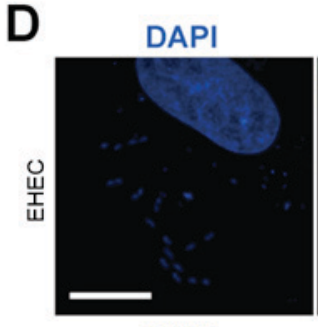

DAPI
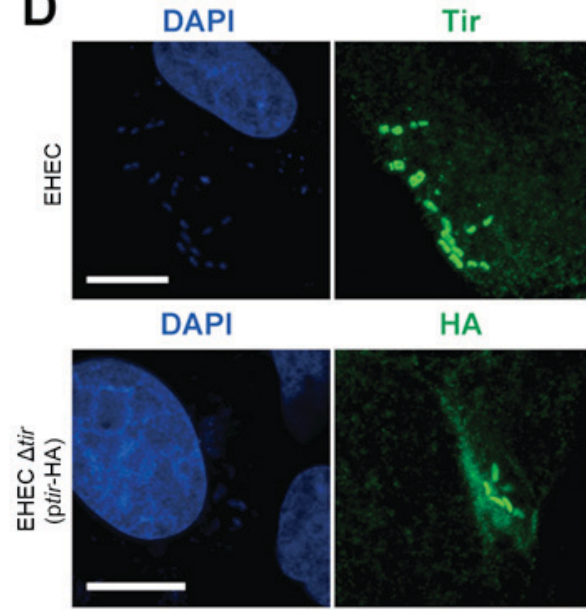

HA
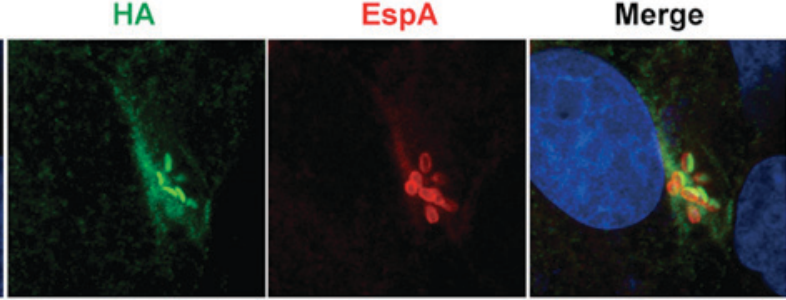

E
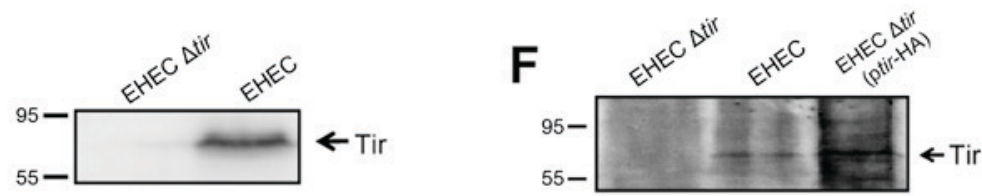

Figure 1. Construction of EHEC $\Delta$ tir mutant and the complemented $\Delta$ tir strain and generation of rabbit anti-reTirC $\mathrm{C}_{405-523}$ antibody. (A) Analysis of purified rabbit anti-reTirC ${ }_{405-523}$ antibody by reducing (left) and non-reducing (right) SDS-PAGE. (B) SDS-PAGE analysis of purified reTirC ${ }_{405-523}$. (C) Identification of the binding of rabbit anti-reTirC $\mathrm{C}_{405-523}$ antibody to reTirC $\mathrm{C}_{405-523}$ by western blotting. (D) Identification of the binding of rabbit anti-reTirC $\mathrm{C}_{405-523}$ antibody to Tir by immunofluorescence assay. (E and F) Identification of tir expression of EHEC $\Delta$ tir mutant and the complemented strain by western blotting using purified rabbit anti-Tir polyclonal antibodies as primary antibody. 
A
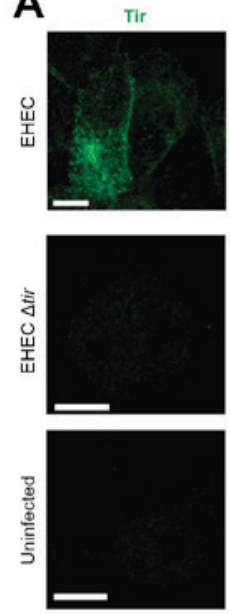

Golgi
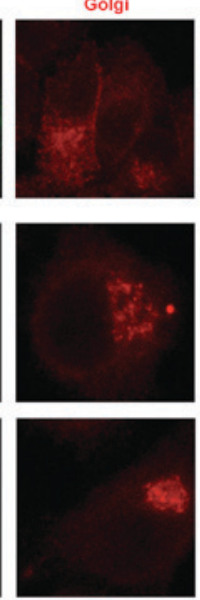

Merge
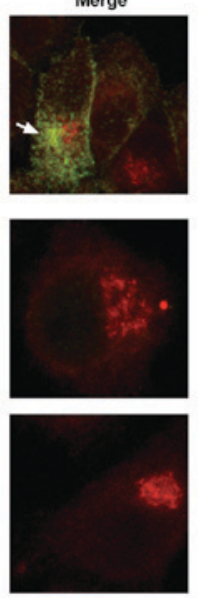

B
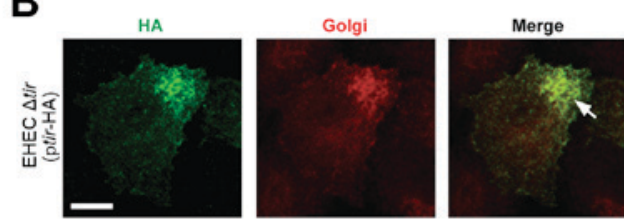

C

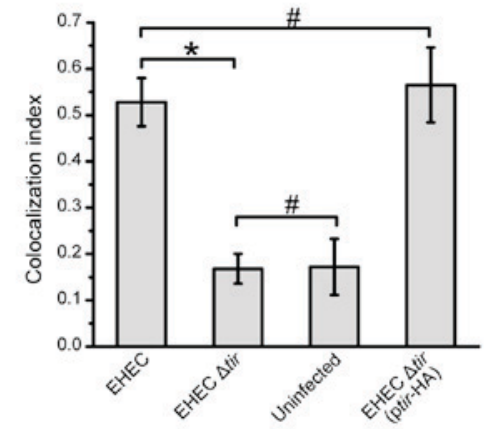

Golgi

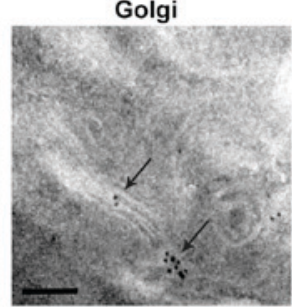

Figure 2. Characterization of the intracellular localization of Tir in HeLa cells. (A and B) Colocalization of Tir and the 58K Golgi protein in HeLa cells. HeLa cells were stained with antibodies against Tir (A) or HA (B) and 58K Golgi protein (A and B). Arrows indicate the colocalization of Tir in Golgi. Scale bar=10 $\mu \mathrm{m}$. (C) Quantitative analysis of colocalization of Tir and 58K Golgi protein. The bar represents the colocalization index of Tir or HA-Tir and 58K Golgi protein, which was estimated by Pearson's correlation coefficient by colocalization finder plugin of ImageJ. Data are representatives of three separate experiments. *indicates $\mathrm{P}<0.05$ and ${ }^{\# \text { indicates } P>0.05}$. (D) Immunoelectron microscopic localization of Tir in HeLa cells. Arrows indicate immunogold-labeled Tir. PM (magnification, x39,000). Golgi (magnification, x65,000). Scale bar=100 nm.

was further quantified. As shown in Fig. 3B, the percentage of EHEC-induced actin pedestals decreased significantly in the presence of BFA $(\mathrm{P}<0.05)$. These results suggest that damage to the Golgi body by BFA blocks the formation of the Tir-mediated actin pedestals.

BFA causes disassembly of the Golgi complex and blocks Tir transportation from Golgi to plasma membrane. To further confirm that BFA-induced damage, the Golgi body was stained in the presence of BFA. Upon treatment with BFA, the $58 \mathrm{~K}$ Golgi proteins diffused throughout the cytoplasm either in the presence or absence of EHEC infection (Fig. 4A, compare to Fig. 2A). Furthermore, Tir was diffusely dispersed in the cytoplasm and around the nuclear envelope, in contrast to its condensed localization in the EHEC-infected cells (Fig. 2A and B). These results indicate that BFA disrupts the structure of the Golgi complex and affects the localization of Tir.

To further determine the role of Golgi body in transporting Tir to plasma membrane, HeLa cells were infected with wild-type EHEC or $\triangle$ tir EHEC complemented with ptir-HA. Western blotting was then used to detect Tir in both cytosolic and membrane fractions. To ensure that both phosphorylated and non-phosphorylated forms in membrane fraction were detected $(12,19)$, the membrane fraction was treated with calf intestinal alkaline phosphatase (Alk Phos), a nonspecific phosphatase before detection. As shown in Fig. 4C, no significant change of Tir distribution in cytosol fraction was observed in cells treated with or without BFA, suggesting that BFA treatment did not affect the release of Tir into HeLa cytoplasm. Tir was also observed in the membrane fractions when cells were infected with either EHEC or $\Delta$ tir EHEC (+ptir-HA). However, when cells were pre-treated with BFA, Tir was not detected in the membrane fraction. These results suggest that BFA blocks the transportation of Tir to plasma membrane.

\section{Discussion}

Bacterial effectors need to be transported to their specific intracellular locations to participate in pathogenesis. Two possible mechanisms of effector transport have been reported (20-22). One is called post-translocation modification of effectors. For example, the cysteine residue at the C-terminus of Salmonella effector SifA is lipidated by the protein geranylgeranyl transferase I, leading to the accumulation of SifA at the PM (20). The second mechanism requires dedicated structural motifs within the effectors. Pseudomonas aeruginosa ExoS and ExoT, and YopE from Yersinia spp. carry a shared membrane localization domain (MLD), which mediates their initial association with the PM $(21,22)$. It has also been reported that the Tir from EPEC $\left(\operatorname{Tir}_{\text {EPEC }}\right.$ ) is transported into the host membrane via two pathways First, the $\operatorname{Tir}_{\text {EPEC }}$ is translocated into the cytoplasm via a type III secretion system, and subsequently integrated into the membrane (23). In the second pathway, 
A
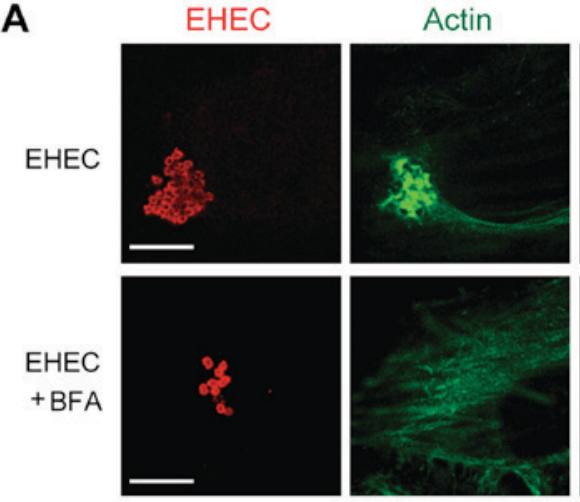

BFA
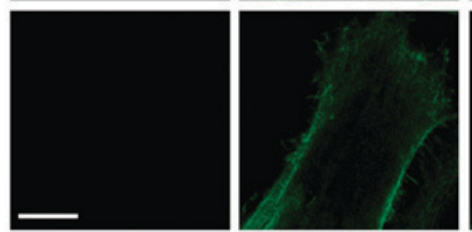

B

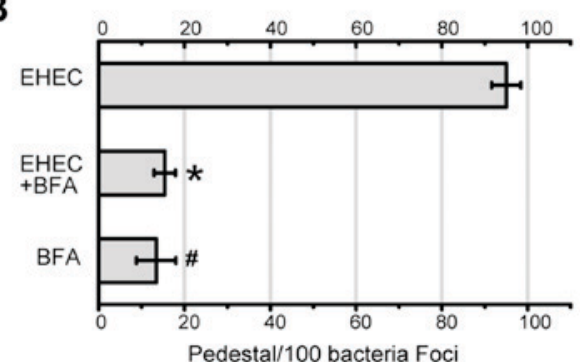

Figure 3. (A) Effect of BFA on EHEC-induced actin pedestal. HeLa cells were stained with antibodies against EspA of EHEC (red) and actin (green), The upper panel shows HeLa cells infected with EHEC; the middle panel shows HeLa cells treated with BFA prior to EHEC infection; and the lower panel shows uninfected HeLa cells treated with BFA. Scale bar $=10 \mu \mathrm{m}$. (B) Quantitative analysis of actin pedestal formation. The bar represents the percentage of actin pedestals associated with the foci of attached bacteria. 'EHEC+BFA' indicates cells were treated with BFA prior to EHEC infection. *indicated $\mathrm{P}<0.05$ when compared with EHEC, ${ }^{*}$ indicated $\mathrm{P}>0.05$ when compared with 'EHEC+BFA' group. Experiments were performed in triplicate.

extracellular Tir directly integrates into host membranes in the presence of limited free $\mathrm{Ca}^{2+}$ in the environment, and the integration is independent of T3SS translocation (24). The Tir of EHEC is highly homologous to $\operatorname{Tir}_{\mathrm{EPEC}}$ and may share a similar pathway for its translocation. In this study, we found that Tir from EHEC was colocalized with the host Golgi structure. Tir was undetected in plasma membrane, when the Golgi structure was disrupted by BFA (Fig. 4C), suggesting that Tir was first transported to cytoplasm/Golgi and then to the cell membrane in a Golgi-dependent manner. This view is further supported by the inhibition of the formation of actin pedestals by BFA, which requires Tir at the plasma membrane. Further studies are needed to understand the mechanisms mediating Tir transport into the cytoplasm (possibly via T3SS) and then to the Golgi apparatus of the host cells.

A possible pathway of Tir transportation to Golgi body may involve the guanine nucleotide-exchange factors (GEFs), which convert the GDP-bound, inactive ADP-ribosylation factor (Arf) to GTP-bound, active Arf (Arf-GTP) $(16,25)$. Arf-GTP associates reversibly with the Golgi membrane to recruit coat proteins and adaptors from the cytosol, and initiates the formation of
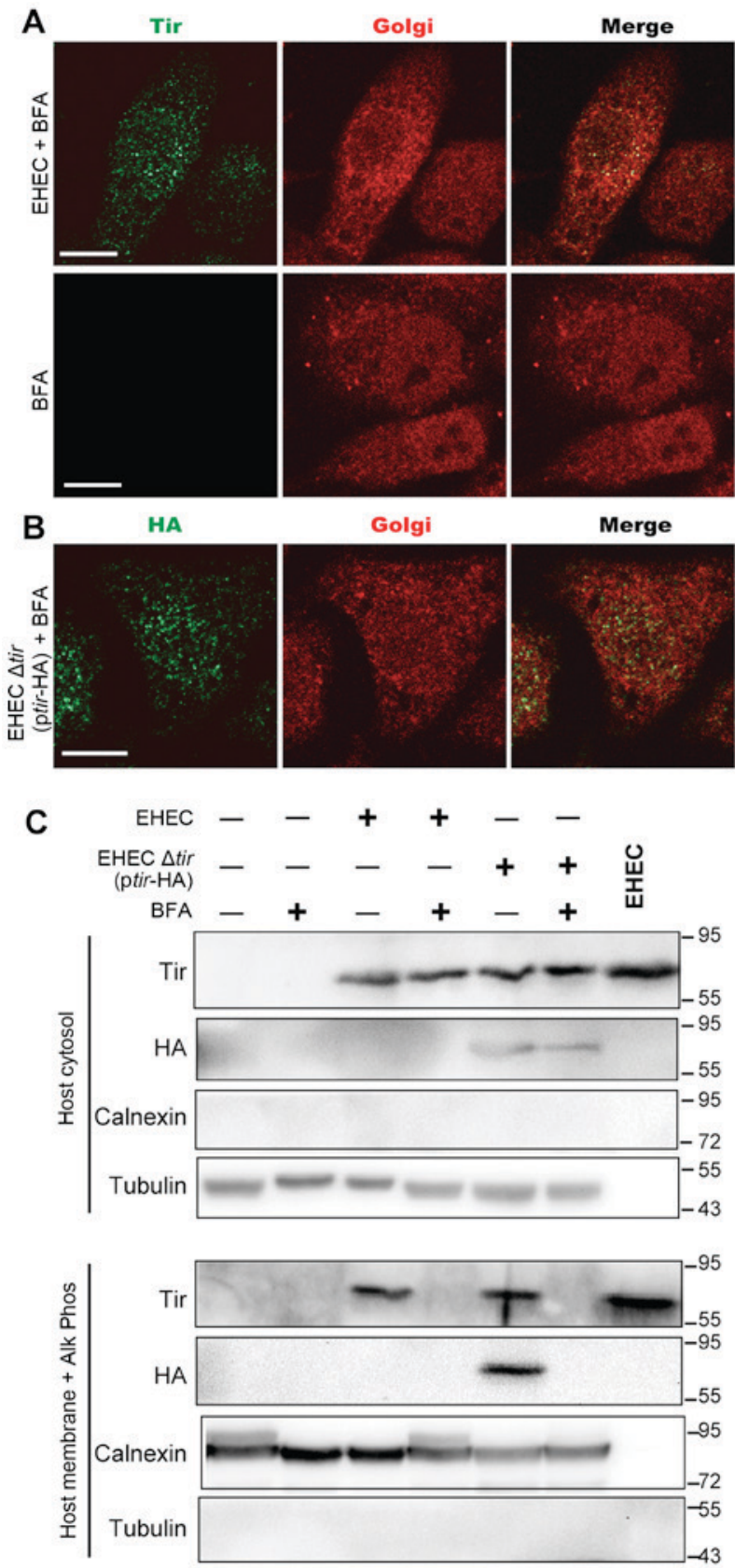

Figure 4. Effect of BFA on Tir translocation to Golgi and its integration into plasma membrane. HeLa cells were pretreated with BFA, and then infected with EHEC or the complemented strain of EHEC $\triangle$ tir. (A and B) BFA caused disassembly of the Golgi (red), and disrupted the translocation of Tir to Golgi (upper panel). Scale bar $=20 \mu \mathrm{m}$. (C) Western blotting of Tir fractions in HeLa cells. HeLa cells were subjected to subcellular fractionation and membrane fractions were treated with alkaline phosphatase (Alk Phos), and probed with anti-Tir, anti-HA, anti-calnexin and anti-tubulin antibodies. Molecular masses $(\mathrm{kDa})$ of standard proteins are indicated. The last column (labeled as EHEC) indicates the cultured bacteria, not the lysed protein of HeLa cells, as the loading sample. Experiments were performed in triplicate.

transport vesicles. BFA prevents the assembly of coatomer on the membrane by inhibiting GEFs, therefore blocking the formation of Arf-GTP (26). This is supported by a study, in which Arf1 accumulates on the Legionella-containing vacuoles and helps the vacuole-ER fusion, which was abolished by BFA (27). Consistent with this report, we observed that Tir was diffusely distributed in the cytoplasm and around the nuclear envelope in the presence of BFA. 
BFA-induced inhibition of Tir transportation is also clear from the endosomal tubulation caused by BFA similar to that induced in the Golgi apparatus (13). Endocytosis is an essential cellular process involved in the transport of membrane proteins in mammalian cells (14). It is reasonable to speculate that endosomes may play a role in Tir transportation to plasma membrane. The role of endosomes in Tir translocation and Tir-induced A/E lesions needs further investigation.

In this study, we report that Tir is translocated to the host plasma membrane in a Golgi-dependent manner. This finding provides a novel trafficking pathway for secreted bacterial effectors. Further studies should be focused on the functional motifs underlying Tir trafficking and its detailed transport mechanism within the Golgi protein delivery system.

\section{Acknowledgements}

We are grateful to Dr. Fuquan Hu for the critical reading and editing of the manuscript. We also thank Wei Sun and Liting Wang for technical assistance with confocal microscopy and George F. Gao (Institute of Microbiology, Chinese Academy of Sciences) for his kind gifts of plasmids pKD3 and pKD46. This study was supported by the National Natural Science Foundation of China (NSFC; no. 81271783).

\section{References}

1. Galán JE: Common themes in the design and function of bacterial effectors. Cell Host Microbe 5: 571-579, 2009.

2. Troisfontaines P and Cornelis GR: Type III secretion: More systems than you think. Physiology (Bethesda) 20: 326-339, 2005.

3. Geissler B: Bacterial toxin effector-membrane targeting: Outside in, then back again. Front Cell Infect Microbiol 2: 75, 2012.

4. Frankel G and Phillips AD: Attaching effacing Escherichia coli and paradigms of Tir-triggered actin polymerization: Getting off the pedestal. Cell Microbiol 10: 549-556, 2008.

5. Campellone KG: Cytoskeleton-modulating effectors of enteropathogenic and enterohaemorrhagic Escherichia coli: Tir, EspFU and actin pedestal assembly. FEBS J 277: 2390-2402, 2010.

6. Campellone KG, Robbins D and Leong JM: EspFU is a translocated EHEC effector that interacts with Tir and N-WASP and promotes Nck-independent actin assembly. Dev Cell 7: 217-228, 2004.

7. Garmendia J, Phillips AD, Carlier MF, Chong Y, Schüller S, Marches O, Dahan S, Oswald E, Shaw RK, Knutton S and Frankel G: TccP is an enterohaemorrhagic Escherichia coli O157: H7 type III effector protein that couples Tir to the actin-cytoskeleton. Cell Microbiol 6: 1167-1183, 2004.

8. Weiss SM, Ladwein M, Schmidt D, Ehinger J, Lommel S, Städing K, Beutling U, Disanza A, Frank R, Jänsch L, et al: IRSp53 links the enterohemorrhagic E. coli effectors Tir and EspFU for actin pedestal formation. Cell Host Microbe 5: 244-258, 2009.

9. Brady MJ, Campellone KG, Ghildiyal $\mathrm{M}$ and Leong JM: Enterohaemorrhagic and enteropathogenic Escherichia coli Tir proteins trigger a common Nck-independent actin assembly pathway. Cell Microbiol 9: 2242-2253, 2007.

10. de Groot JC, Schlüter K, Carius Y, Quedenau C, Vingadassalom D, Faix J, Weiss SM, Reichelt J, Standfuss-Gabisch C, Lesser CF, et al: Structural basis for complex formation between human IRSp53 and the translocated intimin receptor Tir of enterohemorrhagic E. coli. Structure 19: 1294-1306, 2011.
11. Garmendia J, Carlier MF, Egile C, Didry D and Frankel G: Characterization of TccP-mediated N-WASP activation during enterohaemorrhagic Escherichia coli infection. Cell Microbiol 8: 1444-1455, 2006.

12. DeVinney R, Stein M, Reinscheid D, Abe A, Ruschkowski S and Finlay BB: Enterohemorrhagic Escherichia coli O157: H7 produces Tir, which is translocated to the host cell membrane but is not tyrosine phosphorylated. Infect Immun 67: 2389-2398, 1999.

13. Yu S, Gu J, Wang HG, Wang QX, Luo P, Wu C, Zhang WJ, Guo G, Tong WD, Zou QM and Mao XH: Identification of a novel linear epitope on EspA from enterohemorrhagic E. coli using a neutralizing and protective monoclonal antibody. Clin Immunol 138: 77-84, 2011.

14. Datsenko KA and Wanner BL: One-step inactivation of chromosomal genes in Escherichia coli K-12 using PCR products. Proc Natl Acad Sci USA 97: 6640-6645, 2000.

15. Wang H, Gu J, Yu S, Zhang W, Zhu Y, Zou Q, Zhu F and Mao X: Characterization of enterohemorrhagic Escherichia coli $\mathrm{O} 157: \mathrm{H} 7$ 00B015: A Shiga toxin producing but virulence-attenuated isolate. Can J Microbiol 56: 651-656, 2010.

16. Fujiwara T, Oda K, Yokota S, Takatsuki A and Ikehara Y: Brefeldin A causes disassembly of the Golgi complex and accumulation of secretory proteins in the endoplasmic reticulum. $\mathrm{J}$ Biol Chem 263: 18545-18552, 1988.

17. Gruenheid S, Sekirov I, Thomas NA, Deng W, O'Donnell P, Goode D, Li Y, Frey EA, Brown NF, Metalnikov P, et al: Identification and characterization of NleA, a non-LEE-encoded type III translocated virulence factor of enterohaemorrhagic Escherichia coli O157:H7. Mol Microbiol 51: 1233-1249, 2004.

18. Campellone KG, Brady MJ, Alamares JG, Rowe DC, Skehan BM, Tipper DJ and Leong JM: Enterohaemorrhagic Escherichia coli Tir requires a C-terminal 12-residue peptide to initiate EspF-mediated actin assembly and harbours N-terminal sequences that influence pedestal length. Cell Microbiol 8: 1488-1503, 2006.

19. Kenny B: The enterohaemorrhagic Escherichia coli (serotype O157:H7) Tir molecule is not functionally interchangeable for its enteropathogenic E. coli (serotype O127:H6) homologue. Cell Microbiol 3: 499-510, 2001.

20. Reinicke AT, Hutchinson JL, Magee AI, Mastroeni P, Trowsdale J and Kelly AP: A Salmonella typhimurium effector protein SifA is modified by host cell prenylation and S-acylation machinery. J Biol Chem 280: 14620-14627, 2005.

21. Kazmierczak BI and Engel JN: Pseudomonas aeruginosa ExoT acts in vivo as a GTPase-activating protein for RhoA, Rac1, and Cdc42. Infect Immun 70: 2198-2205, 2002.

22. Black DS and Bliska JB: The RhoGAP activity of the Yersinia pseudotuberculosis cytotoxin YopE is required for antiphagocytic function and virulence. Mol Microbiol 37: 515-527, 2000.

23. Kenny B, DeVinney R, Stein M, Reinscheid DJ, Frey EA and Finlay BB: Enteropathogenic E. coli (EPEC) transfers its receptor for intimate adherence into mammalian cells. Cell 91: 511-520, 1997.

24. Michgehl S, Heusipp G, Greune L, Rüter C and Schmidt MA: Esp-independent functional integration of the translocated intimin receptor (Tir) of enteropathogenic Escherichia coli (EPEC) into host cell membranes. Cell Microbiol 8: 625-633, 2006.

25. Klausner RD, Donaldson JG and Lippincott-Schwartz J: Brefeldin A: Insights into the control of membrane traffic and organelle structure. J Cell Biol 116: 1071-1080, 1992.

26. Donaldson JG, Finazzi D and Klausner RD: Brefeldin A inhibits Golgi membrane-catalysed exchange of guanine nucleotide onto ARF protein. Nature 360: 350-352, 1992.

27. Kagan JC and Roy CR: Legionella phagosomes intercept vesicular traffic from endoplasmic reticulum exit sites. Nat Cell Biol 4: 945-954, 2002. 